BNL-101037-2013-IR

\title{
Analysis of localized beam losses in the Booster extraction straight section and the Booster to Storage Ring transfer line
}

\author{
S. Seletskiy
}

June 5, 2013

Photon Sciences Directorate

Brookhaven National Laboratory

\section{U.S. Department of Energy DOE - Office of Science}

Notice: This manuscript has been authored by employees of Brookhaven Science Associates, LLC under Contract No. DE-AC02-98CH10886 with the U.S. Department of Energy. The publisher by accepting the manuscript for publication acknowledges that the United States Government retains a non-exclusive, paid-up, irrevocable, world-wide license to publish or reproduce the published form of this manuscript, or allow others to do so, for United States Government purposes.

This preprint is intended for publication in a journal or proceedings. Since changes may be made before publication, it may not be cited or reproduced without the author's permission. 


\section{DISCLAIMER}

This report was prepared as an account of work sponsored by an agency of the United States Government. Neither the United States Government nor any agency thereof, nor any of their employees, nor any of their contractors, subcontractors, or their employees, makes any warranty, express or implied, or assumes any legal liability or responsibility for the accuracy, completeness, or any third party's use or the results of such use of any information, apparatus, product, or process disclosed, or represents that its use would not infringe privately owned rights. Reference herein to any specific commercial product, process, or service by trade name, trademark, manufacturer, or otherwise, does not necessarily constitute or imply its endorsement, recommendation, or favoring by the United States Government or any agency thereof or its contractors or subcontractors. The views and opinions of authors expressed herein do not necessarily state or reflect those of the United States Government or any agency thereof. 


\section{Introduction}

The NSLS-II accelerators are installed within radiation shielding walls that are designed to attenuate the radiation generated from an assumed beam loss power (current multiplied by the assumed energy) within the beam channel to a level of $<0.5 \mathrm{mrem} / \mathrm{h}$ at the outer surface of the bulk shield walls of the accelerator. This Bulk Shielding Power assumes that the beam current is intercepted by a thick target (i.e., $30 \mathrm{~cm}$ of copper or steel) along the Booster or transport line beam axis and the dose rate level is calculated transverse to the beam axis. Any operational losses greater than this BSP level are expected to be addressed by installing supplemental shielding near that loss point in order to attenuate this radiation level outside the shield wall to the design level of $<0.5 \mathrm{mrem} / \mathrm{hr}$.

The purpose of this exercise is to determine the potential beam loss locations, for further identification of the radiation risks they impose outside the existing bulk shielding, and specification of the supplemental shielding design necessary to mitigate those risks.

We will present the beam systems description along with their planned operating ranges, which forms the basis of our analysis. Next, we will describe the method used for each system to determine potential beam loss locations. Finally, as a conclusion, we will present the results of our studies in a form of convenient to use table which includes all the information necessary for analysis of radiation risks induced by the determined beam losses.

In the following analysis we assume, unless the opposite is indicated in the clearest possible terms, that the accelerator is configured in any feasible way that its design allows.

\section{Description of the beamlines}

We consider three beamlines: the Booster extraction straight section (ES), the Booster to Storage Ring (BSR) transfer line phase 1 and the Booster to Storage Ring transfer line phase 2. Figure 1 (obtained from [1]) shows the beamlines under consideration.

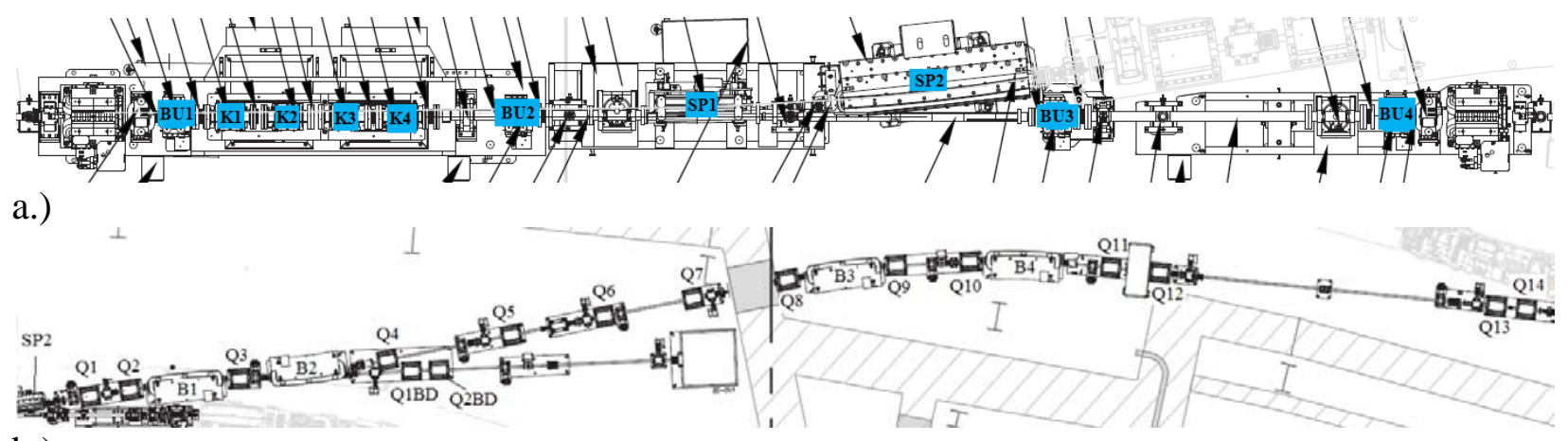

b.)

Figure 1: Schematics of ES (a) and BSR phase 1 and phase 2 (b).

The extraction section consists of four slow bumps forming local closed 4-bump BU1, BU2, BU3 and BU4 (their respective lattice file names are BRBU1SE, BRBU2SE, BRBU3SE and BRBU4SE; their respective engineering names are BR-XSBUM1, BR-XSBUM2, BR-XSBUM3 and BR-XSBUM4), four fast kickers K1, K2, K3 and K4 (their respective lattice file names are 
BRK1SE, BRK2SE, BRK3SE and BRK4SE; their respective engineering names are BRXSKIC1-1, BR- XSKIC1-2, BR- XSKIC2-1 and XSKIC2-2), pulsed septum magnet SP1 (its lattice file name is BRSP1SE and its engineering name is BR-XSSMP1), and DC septum magnet SP2 (its engineering name is BR-XSSMD1). These magnets are utilized for the extraction of the beam from the Booster into the BSR line.

The phase 1 of BSR line (a.k.a. dump line) consists of five quadrupoles Q1, Q2, Q3, Q1BD and Q2BD (their respective engineering names are BS-Q1, BS-Q2, BS-Q3, BS-Q1BD and BS$\mathrm{Q} 2 \mathrm{BD}$ ), and one bending magnet B1 (its engineering name is BS-B1). This magnets transport beam to the beam dump when bending magnet $\mathrm{B} 2$ is turned off.

The BSR line phase 2 includes magnets Q1, Q2, Q3 and B1, and additionally has quadrupoles Q4, Q5, Q6, Q7, Q8, Q9, Q11, Q12, Q13, Q14 (BS-Q4, BS-Q5, BS-Q6, BS-Q7, BS-Q8, BS-Q9, BS-Q11, BS-Q12, BS-Q13, BS-Q14 in engineering notation) and bends B2, B3 and B4 (BS-B2, BS-B3, BS-B4 in engineering notation). This magnets transport beam to the Storage Ring. Injection into the Storage Ring is performed with SP3 and IS, which are the DC and pulsed Storage Ring injection septa respectively. Their engineering names are BS-SP3 and SR-IS-SP1.

\section{Description of beamline elements}

Each of the four bumps BU1-BU4 is an "H"-shape magnet consisting of a yoke and two coils [2]. The bumps nominal bending angle at $3 \mathrm{GeV}$ is $7.5 \mathrm{mrad}$. All four bumps are powered in series. The peak current fed to the magnet is $1500 \mathrm{~A}$ (the power supply can provide about $10 \%$ higher current). The pulse length is $3 \mathrm{~ms}$ for one sinusoid. The bump's horizontal gap (horizontal distance between the yokes material) is about $152 \mathrm{~mm}$. Figure 2 shows the general view of the bump magnet.
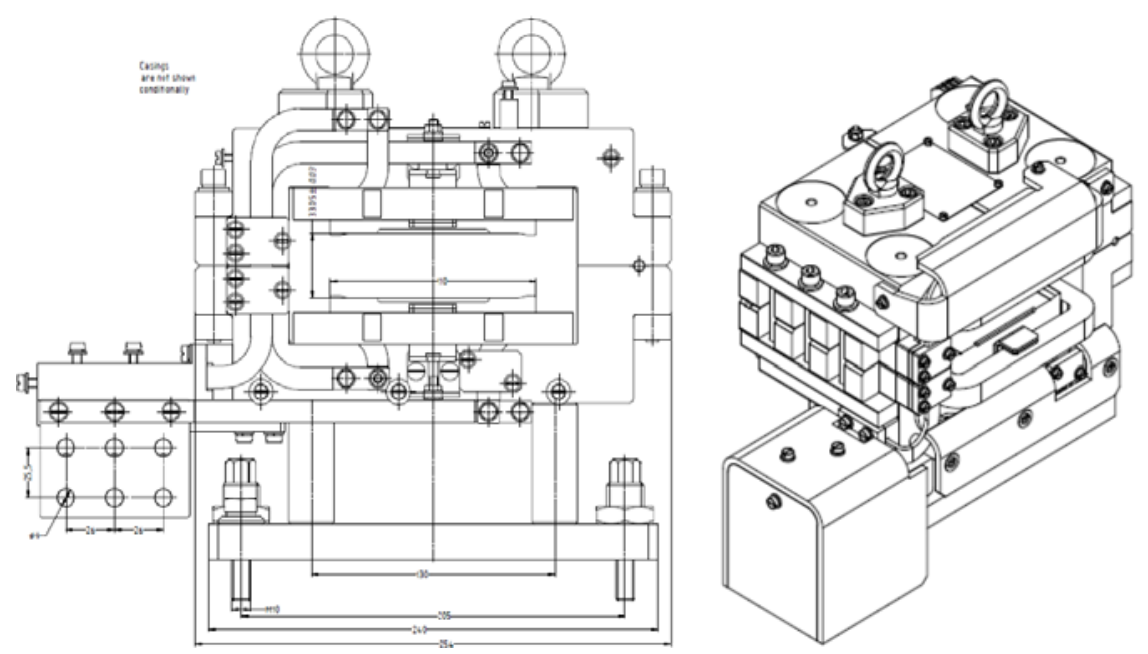

Figure 2: The central cross-section (left) and a general view of the bump magnet (right).

The fast kicker [2] is shown in Figure 3. The kickers provide nominal bending angle at 3 $\mathrm{GeV}$ of 1.45 mrad each. The kicker's pulse has $215 \mathrm{nsec}$ rise time and 310 nsec flat-top. The flat top current is $2.3 \mathrm{kA}$. The power supply (PS) at maximum can provide about $10 \%$ higher current. Kickers K1 and K2 are powered in series. Kickers K3 and K4 are powered in series. Each kicker's horizontal gap is $60 \mathrm{~mm}$. 

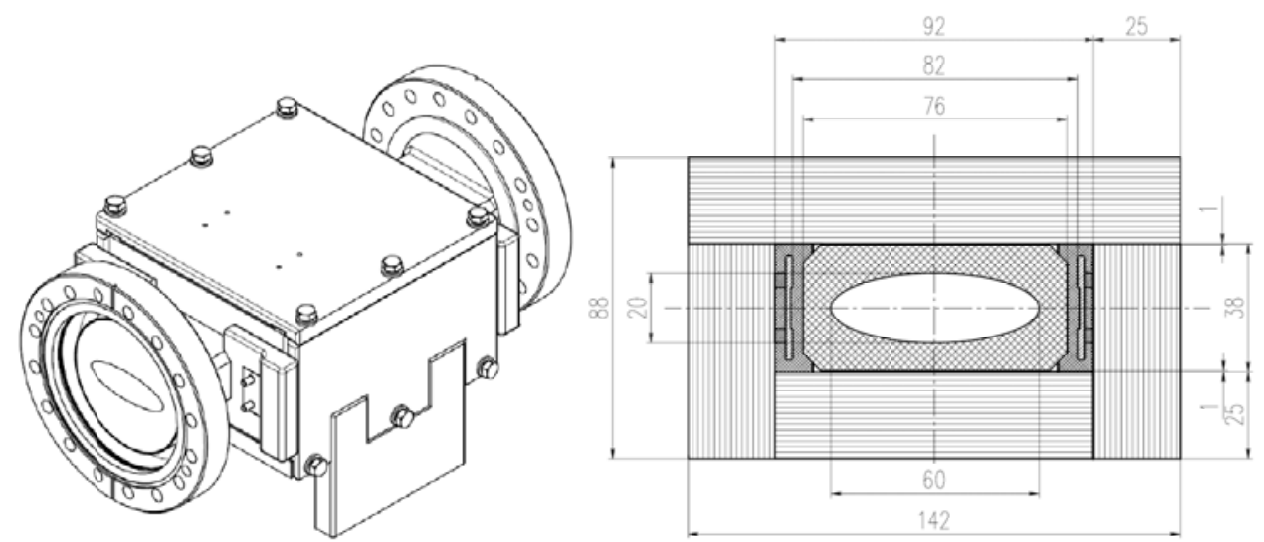

Figure 3: A general view (left) and the central cross-section of the kicker magnet.

The pulsed septum has a nominal bending angle of 48mrad [2]. The nominal current at the peak field from pulsed septum PS is $10.22 \mathrm{kA}$ (it can be about $10 \%$ higher). The extraction channel is $18 \mathrm{~mm}$ x $16 \mathrm{~mm}$ (horizontal x vertical) and accommodates the thin-walled $(0.3 \mathrm{~mm})$ stainless steel vacuum chamber of $15 \mathrm{~mm}$ diameter. Figure 4 shows the cross-section and the top view of the pulsed septum.

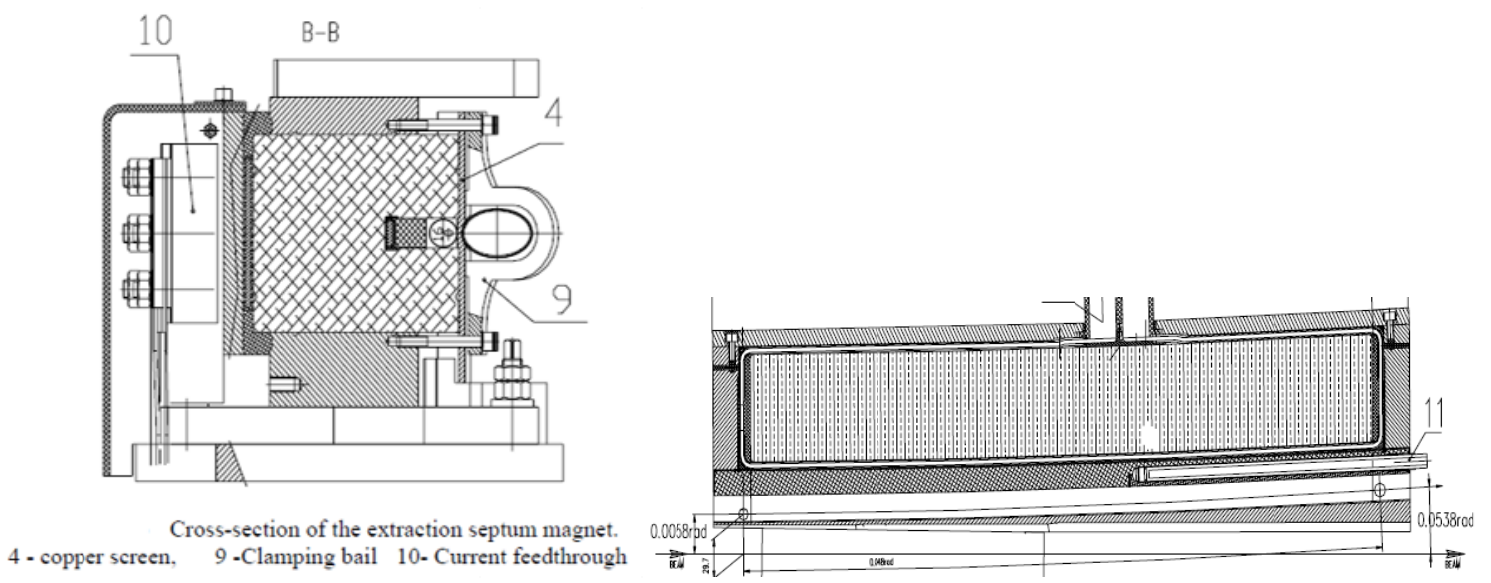

Figure 4: The cross-section (left) and the top view (right) of the pulsed extraction septum.

The DC septum is a "C"-shape bending magnet. The nominal bending angle of the DC septum is $104 \mathrm{mrad}$ [3]. A nominal power supply current is $440 \mathrm{~A}$ and the maximum current is 500 A [4]. The DC septum accommodates vacuum chamber of $30 \mathrm{~mm}$ diameter. The distance from the reference trajectory to the septum yoke is about $140 \mathrm{~mm}$. The view of the DC septum is given in Figure 5. 


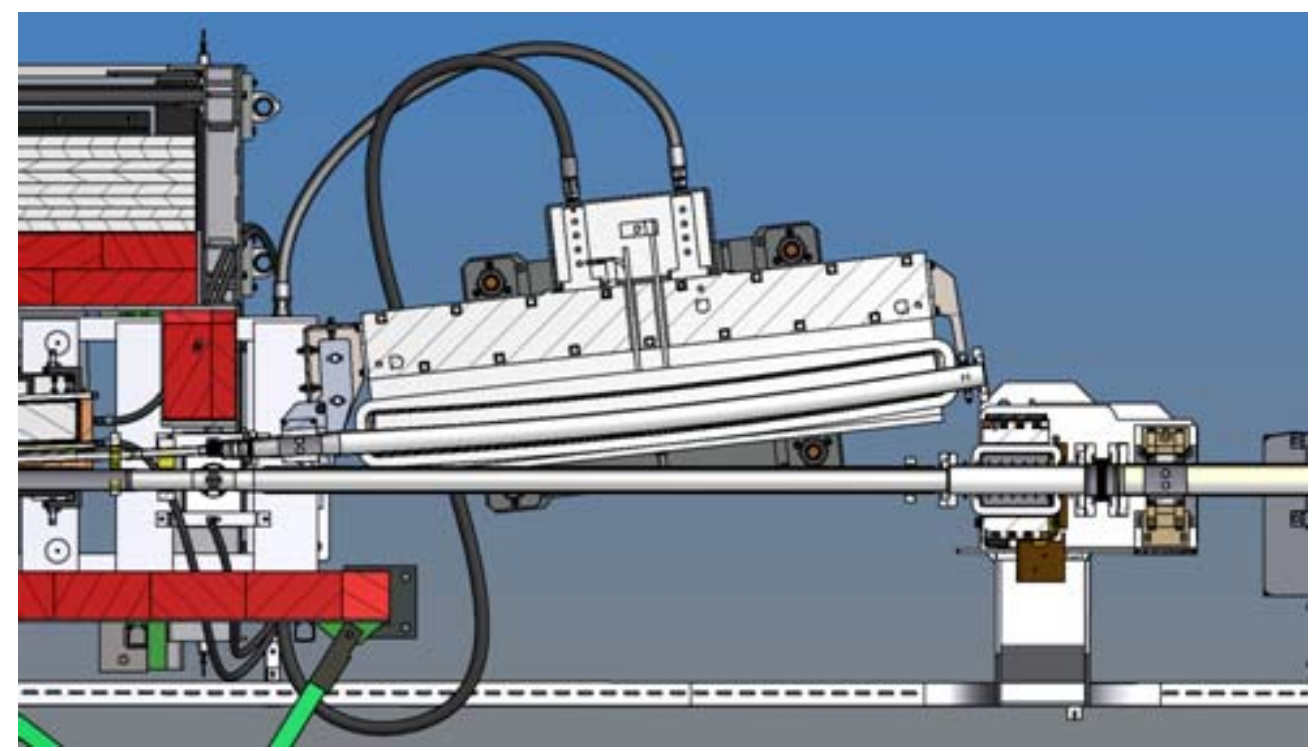

Figure 5: The top view of the DC septum.

The DC injection septum (SP3) is identical to SP2. Its nominal bending angle is 114 mrad.

The pulsed injection septum (IS) is a C-shaped magnet. It accommodates the vacuum chamber with $21 \mathrm{~mm}$ horizontal aperture. The septum yoke is touching the vacuum chamber. The nominal bending angle of pulsed septum is $100 \mathrm{mrad}$. The nominal current at the peak field from pulsed septum PS is $7.9 \mathrm{kA}$ (it can be about $10 \%$ higher).

The four BSR bends (B1-B4) are identical "C"-shape magnets. Their nominal bending angles are given in Table 5. Bends power supplies can provide $375 \mathrm{~A}$ at maximum [4]. The measurement of B1 gives the conversion coefficient of $0.00502 \mathrm{Tm} / \mathrm{A}$ [5]. Bends accommodate vacuum chamber of $120 \mathrm{~mm}$ width [6]. The distance from the reference trajectory to the yoke of the bend is about $220 \mathrm{~mm}$. The top view of the DC septum is shown in Figure 6.

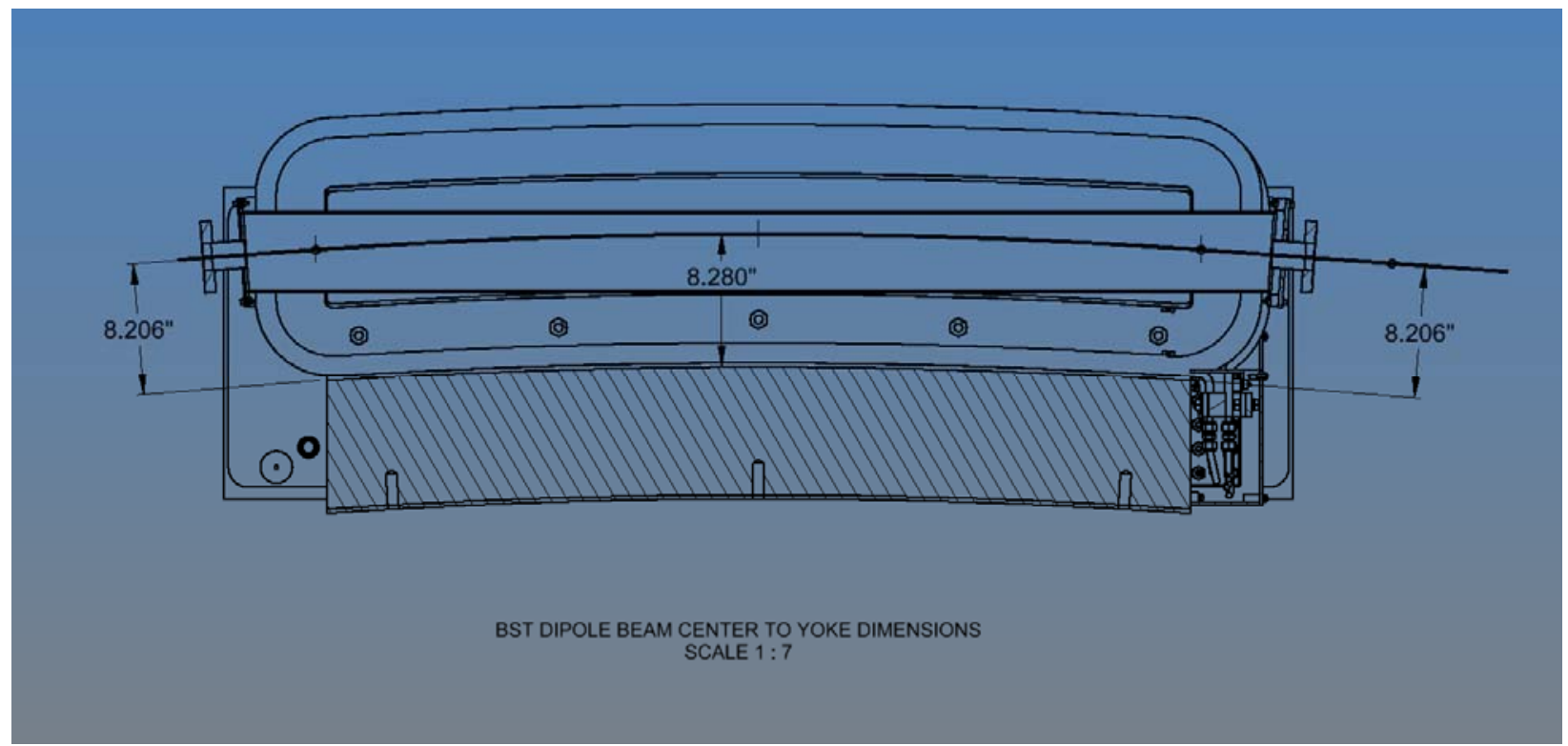

Figure 6: Top view of the DC septum (courtesy of M. Johansen) 
The sixteen BSR quadrupoles (Q1-Q14, Q1BD and Q2BD) are identical. Their nominal gradients are given in Table 5. The maximum currents for quads power supplies are given in Table 1 [4]. The measured conversion coefficients for the quads are shown in Table 2 [5] (we use median value of $0.171 \mathrm{~T} / \mathrm{m} / \mathrm{A}$ for quads Q12-Q14 that were not measured). The quadrupoles accommodate vacuum chamber of $38 \mathrm{~mm}$ diameter [6].

\begin{tabular}{|c|r|r|r|r|r|r|r|r|}
\hline Magnet Name & BSR-Q1 & BSR-Q2 & BSR-Q3 & BSR-Q4 & BSR-Q5 & BSR-Q6 & BSR-Q7 & BSR-Q8 \\
\hline PS Max Current & 175 & 200 & 175 & 175 & 200 & 200 & 175 & 200 \\
\hline Magnet Name & BSR-Q9 & BSR-Q10 & BSR-Q11 & BSR-Q12 & BSR-Q13 & BSR-Q14 & BSR-Q1BD & BSR-Q2BD \\
\hline PS Max Current & 175 & 175 & 200 & 175 & 200 & 175 & 175 & 175 \\
\hline
\end{tabular}

Table 1: The maximum currents of BSR quads power supplies (courtesy of G. Ganetis).

\begin{tabular}{|c|c|c|c|c|c|c|c|c|}
\hline Magnet Name & BSR-Q1 & BSR-Q2 & BSR-Q3 & BSR-Q4 & BSR-Q5 & BSR-Q6 & BSR-Q7 & BSR-Q8 \\
\hline $\mathrm{Q}$ coeff $[\mathrm{T} / \mathrm{m} / \mathrm{A}]$ & 0.171 & 0.170 & 0.171 & 0.171 & 0.171 & 0.158 & 0.163 & 0.171 \\
\hline Magnet Name & BSR-Q9 & BSR-Q10 & BSR-Q11 & BSR-Q1BD & BSR-Q2BD & & & \\
\hline $\mathrm{Q} \operatorname{coeff}[\mathrm{T} / \mathrm{m} / \mathrm{A}]$ & 0.171 & 0.170 & 0.173 & 0.171 & 0.170 & & & \\
\hline
\end{tabular}

Table 2: Conversion coefficients for BSR quads (courtesy of R. Fliller).

\section{Analysis of beam angles in the Booster extraction straight section}

Although the booster ES magnets are supposed to be used only at extraction energy of $3 \mathrm{GeV}$ ( $2 \mathrm{GeV}$ extraction might be intentionally used as well), technically they can fire at any point of Booster ramp. Since these magnets are powered in series there are three scenarios to consider: either kickers K1 and K2 fire, or K3 and K4 fire, or four bumps BU1-4 fire. If the phase space available to the beam centroids at the entrance to the first of the chained magnets is defined by geometric acceptance of upstream drift (see below), and if BU2-4 are treated independently from $\mathrm{BU} 1$, then the three described scenarios cover all possible local beam losses that can happen under any feasible ES settings.

\subsection{Beam angles analysis at low energy}

Since at low energy $(150 \mathrm{MeV})$ electron beam might be a freshly injected one rather than the stored one, the proper method of defining the beam centroids phase space at the entrance of the magnets is to define it by the acceptance of the respective upstream drift. This technique, although it results in overestimates of the lost beam angles provides a bullet-proof guarantee that all possible beams entering the magnet are considered. The described method (below we will refer to it as a "transfer line approach") is illustrated in Figure 7.

Starting with the beam centroids phase space described above we track each beam centroid through the first chained magnet. We stop tracking the beam if it hits the magnet yoke and record the angle at which such beam was "lost". Here, by the lost beam we mean that it is not a bright electron beam traveling towards the concrete wall anymore. 

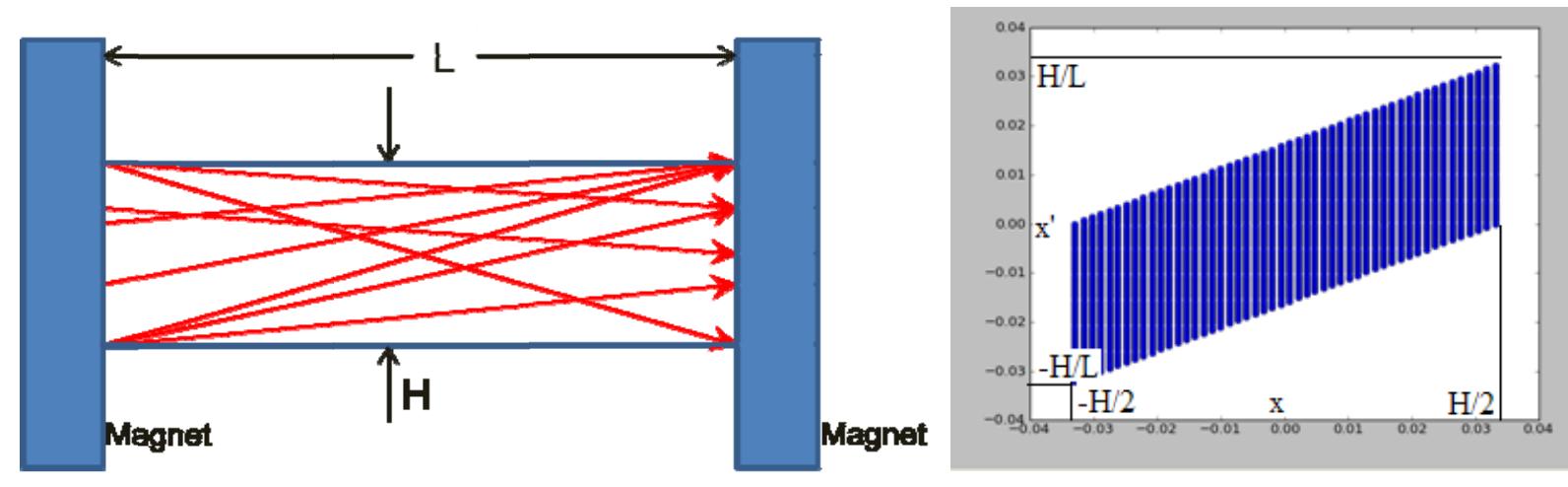

Figure 7: The picture on the left schematically shows the beam centroids (red) originating at the previous magnet and filling the drift upstream of the magnet under consideration. The plot on the right shows the phase space available to the beam centroids at the entrance of the considered magnet.

Next, we record the beam angle at the exit of the magnet and track the beam through the drift to the next chained magnet. If the beam makes it to the entrance of the next magnet without hitting the vacuum chamber, then the described tracking procedure is repeated for this magnet. This routine is continued until the exit of the last chained magnet is reached. Figure 8 illustrates this procedure.

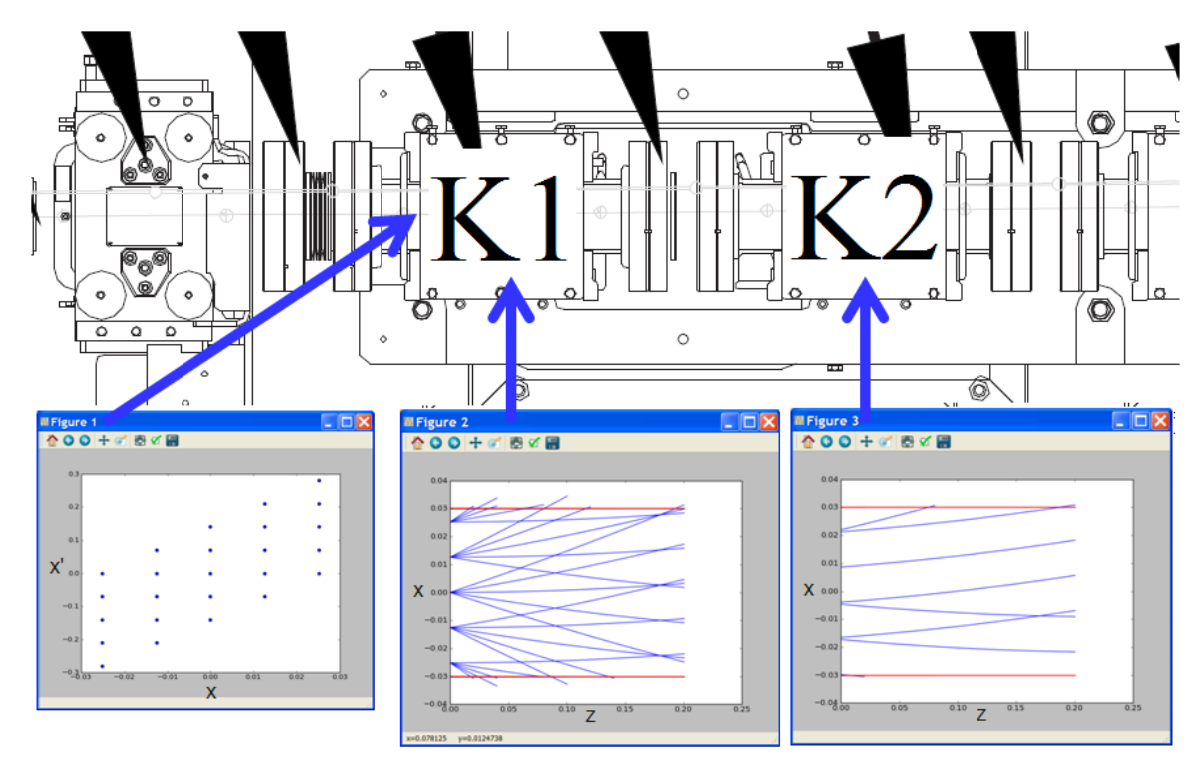

Figure 8: The left plot shows the phase space at the entrance of $\mathrm{K} 1$, which is defined by the geometry of upstream drift, filled with 25 beam centroids. The central plot shows beams tracked through K1, and the right plot shows beams (which survived the $\mathrm{K} 1$ and K1-K2 drift) tracked through K2. Red lines represent magnet yoke. The tracking is shown for maximum K1-2 current only. 


\begin{tabular}{|l|rr|rrrr|}
\hline $\begin{array}{l}\text { element } \\
\text { name }\end{array}$ & $\begin{array}{l}\text { Anom } \\
\text { [rad] }\end{array}$ & $\begin{array}{l}\text { bending } \\
\text { direction }\end{array}$ & $\begin{array}{l}\theta \max \mathrm{R} \\
\text { [rad] }\end{array}$ & $\begin{array}{l}\theta \max \mathrm{L} \\
\text { [rad] }\end{array}$ & $\begin{array}{l}\text { inside } \\
\text { [rad] }\end{array}$ & $\begin{array}{l}\text { lnax } \\
\text { inside } \\
\text { [rad] }\end{array}$ \\
\hline BRBU1SE & 0.0075 & $\mathrm{~L}$ & 0.18311 & 0.33311 & no hits & no hits \\
BRK1SE & 0.00145 & $\mathrm{~L}$ & 0.144824 & 0.167069 & 0.279992 & 0.283182 \\
BRK2SE & 0.00145 & $\mathrm{~L}$ & 0.067584 & 0.112075 & 0.096549 & 0.115519 \\
BRK3SE & 0.00145 & $\mathrm{~L}$ & 0.15959 & 0.179214 & 0.356008 & 0.356008 \\
BRK4SE & 0.00145 & $\mathrm{~L}$ & 0.073657 & 0.112905 & 0.110485 & 0.127403 \\
BRBU2SE & 0.0075 & $\mathrm{R}$ & 0.278052 & 0.113052 & no hits & no hits \\
BRBU3SE & 0.0075 & $\mathrm{R}$ & 0.150948 & 0.011695 & no hits & no hits \\
BRBU4SE & 0.0075 & $\mathrm{~L}$ & 0.007797 & 0.009882 & no hits & no hits \\
\hline
\end{tabular}

Table 3: Maximum angles of lost beams and beams exiting the ES magnets at $150 \mathrm{MeV}$. The $4^{\text {th }}$ and the $5^{\text {th }}$ columns give angles at magnet exit. The $6^{\text {th }}$ and the $7^{\text {th }}$ columns give angles of the beams lost inside the magnet. " $L$ " and "R" stand for left and right bending direction.

The described tracking is performed for each beam centroid (we fill the initial phase space with 2500 centroids). The currents of the chained magnets are scanned in the full range of respective PS.

Finally the maximum angles of the beams lost inside and exiting each magnet are determined. We are interested in both the horizontal angle to the left and angle to the right of beam direction of motion. Results of this analysis are presented in the Table 3.

\subsection{Beam angles analysis at high energy}

At high energy (here we consider $2 \mathrm{GeV}$ and $3 \mathrm{GeV}$ beams) the electron beam is stored in the Booster. This shrinks the phase space available to the beam centroids at the entrance to ES magnets significantly. Indeed, prior to the moment the fast magnets are turned on the stored beam in the ES shall be within the acceptance of $8.15 \mathrm{~m}$ long drift. Therefore, when the magnet is switched on the borders of the phase space at its entrance are defined by the following equation:

$$
\begin{aligned}
& \theta_{\max 1}(h)=\frac{\frac{H}{2}+h}{L_{1}},-H / 2 \leq h \leq-H\left(1 / 2-L_{1} / L\right) \\
& \theta_{\max 2}(h)=\frac{\frac{H}{2}-h}{L-L_{1}},-H\left(1 / 2-L_{1} / L\right) \leq h \leq H / 2 \\
& \theta_{\min 1}(h)=\frac{\frac{H}{2}+h}{L-L_{1}},-H / 2 \leq h \leq H\left(1 / 2-L_{1} / L\right) \\
& \theta_{\min 2}(h)=\frac{\frac{H}{2}-h}{L_{1}}, H\left(1 / 2-L_{1} / L\right) \leq h \leq H / 2
\end{aligned}
$$

The meaning of $H, h, L$ and $L_{1}$ is illustrated in Figure 9.

Although ES bumps are slow in comparison to beam revolution time, the phase space defined by eq. 1 can be immediately used at BU1 entrance since the four bumps are designed to create only the localized distortion of the stored beam trajectory. 

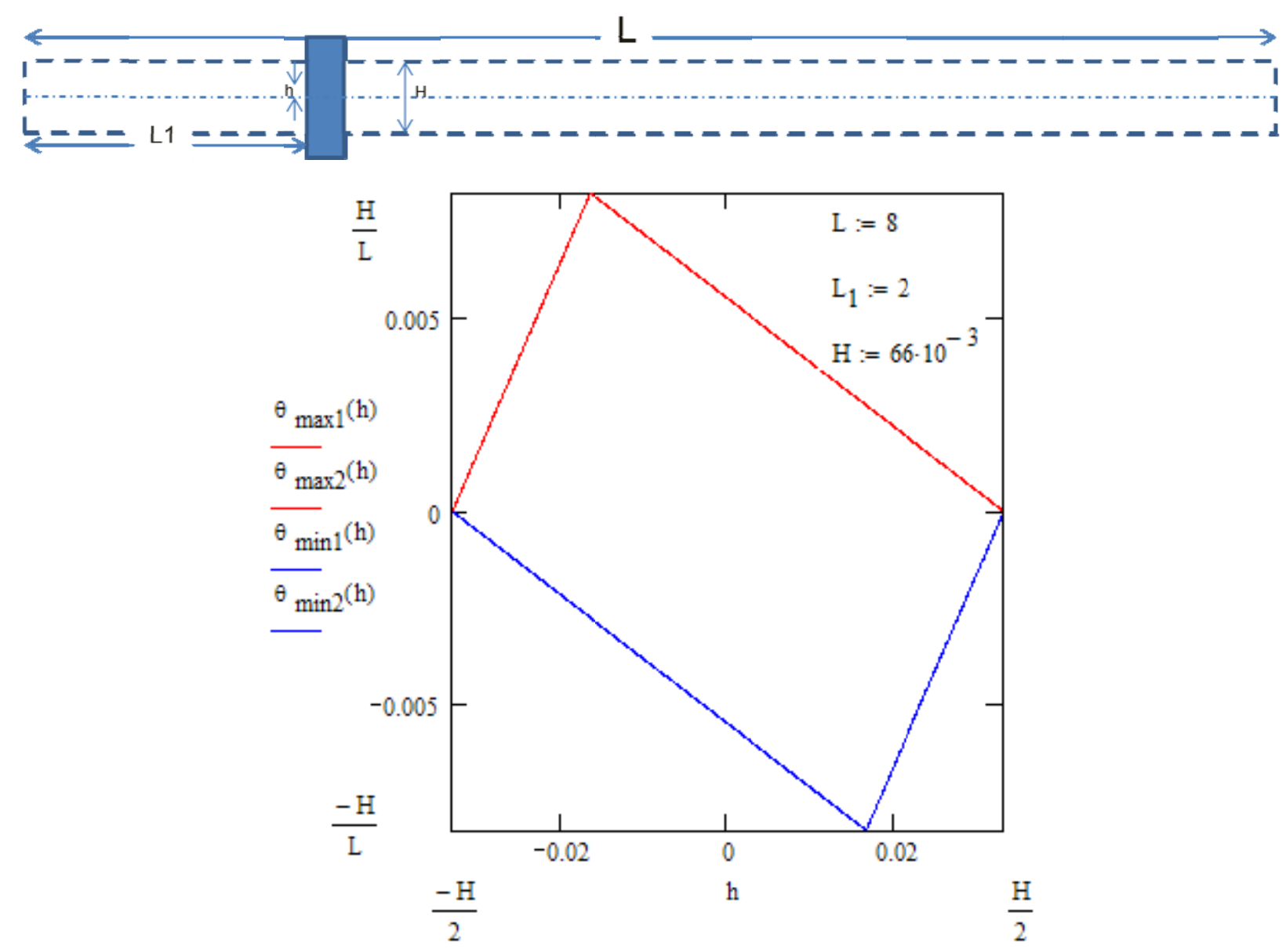

Figure 9: The schematic of ES drift and the phase space in front of the ES magnet for the stored beam.

For an entrance to $\mathrm{K} 1$ we use the phase space described above increased in positive angles by the maximum value available from BU1 kick. Similarly, for K3 the respective phase space shall be increased by maximum summed kicks of BU1, K1 and K2, and for BU2 entrance, the phase space must be increased by the sum of maximum kicks of BU1 and K1-K4.

Such approach significantly reduces the initial phase space available to beam centroids as compared to transfer line approach, while guaranteeing that all possible beams were taken into account.

The rest of the tracking procedure for high energy beams is identical to the one described for $150 \mathrm{MeV}$ beams. The results of beam angles analysis are given in Table 4. 


\begin{tabular}{|c|c|c|c|c|c|c|c|c|c|c|}
\hline $\begin{array}{l}\text { element } \\
\text { name }\end{array}$ & $\begin{array}{l}\theta \text { nom } \\
{[\mathrm{rad}]}\end{array}$ & $\begin{array}{l}\text { bending } \\
\text { direction }\end{array}$ & $\begin{array}{l}\theta \max R \\
{[\mathrm{rad}]}\end{array}$ & $\begin{array}{l}\theta \max L \\
{[\mathrm{rad}]}\end{array}$ & $\begin{array}{l}\text { beam } \\
\text { Omax R } \\
\text { inside } \\
\text { [rad] }\end{array}$ & $\begin{array}{l}\theta \max L \\
\text { inside } \\
\text { [rad] }\end{array}$ & $\begin{array}{l}\theta \max R \\
{[\mathrm{rad}]}\end{array}$ & $\begin{array}{l}\theta \max \mathrm{L} \\
{[\mathrm{rad}]}\end{array}$ & $\begin{array}{l}\text { / beam } \\
\text { Omax R } \\
\text { inside } \\
\text { [rad] }\end{array}$ & $\begin{array}{l}\theta \max L \\
\text { inside } \\
\text { [rad] }\end{array}$ \\
\hline BRBU1SE & 0.0075 & & 0.008098 & 30.020473 & no hits & no hits & 0.008098 & 0.016348 & no hits & no hits \\
\hline BRK1SE & 0.00145 & ; & 0.008098 & 0.022866 & 0.008098 & 0.022866 & 0.008098 & 0.017943 & 0.008098 & 0.017305 \\
\hline BRK2SE & 0.00145 & & 0.008098 & $3 \quad 0.025258$ & 0.008098 & 0.024301 & 0.008098 & 0.019538 & $\begin{array}{ll}3 & 0.008098\end{array}$ & 0.0189 \\
\hline BRK3SE & 0.00145 & & 0.008098 & 0.027651 & 0.008098 & 0.027172 & 0.008098 & 0.021133 & 0.008098 & 0.021133 \\
\hline BRK4SE & 0.00145 & & 0.008098 & 30.030043 & 0.008098 & 0.030043 & 0.008098 & 0.022728 & 30.008098 & 0.022409 \\
\hline BRBU2SE & 0.0075 & ; & 0.020473 & 0.032518 & no hits & no hits & 0.016348 & 0.022728 & no hits & no hits \\
\hline BRBU3SE & 0.0075 & & 0.030218 & 30.020526 & no hits & no hits & 0.024598 & 0.017413 & no hits & no hits \\
\hline BRBU4SE & 0.0075 & ; & 0.010573 & 0.015038 & no hits & no hits & 0.010335 & 0.014478 & no hits & no hits \\
\hline
\end{tabular}

Table 4: Maximum angles of lost beams and beams exiting the ES magnets at $2 \mathrm{GeV}$ and $3 \mathrm{GeV}$. The $4^{\text {th }}, 5^{\text {th }}, 8^{\text {th }}$ and $9^{\text {th }}$ columns give angles at magnet exit. The $6^{\text {th }}, 7^{\text {th }}, 10^{\text {th }}$ and $11^{\text {th }}$ columns give angles of the beams lost inside the magnet. " $L$ " and " $R$ " stand for left and right bending direction.

\section{Analysis of beam angles in the Booster to Storage Ring transfer line}

To determine the beam centroids phase space at the entrances of elements of both phases of BSR line we adapt the transfer line approach described in section 4.1. Next, we track all the beam centroids through respective element. Each beam centroid is tracked through the magnet at its nominal, maximum and minimum possible settings. Finally, we determine the maximum angles of the beams lost inside the magnet as well as the maximum horizontal angles of the beams exiting the magnet both to the left and to the right direction with respect to the direction of beam motion.

For the dipole magnet, the uniform field is used in tracking, which gives a conservative estimate of angles in the bending direction. In the direction opposite to the bending the maximum angle occurs for the switched off magnet.

For the quadrupole the beam hitting the vacuum chamber is considered to be lost, since the space around the chamber is closely packed with material of quad poles and windings.

In the following calculations we assume that the quadrupoles can switch polarity and that the bends always have designed polarity.

The described routine is performed at two energies - at $2 \mathrm{GeV}$ and at $3 \mathrm{GeV}$. The lowest energy at which one might intentionally extract the beam into BSR dump line is $2 \mathrm{GeV}$. Apparently, the maximum angles of beams are obtained at that energy. We also perform $3 \mathrm{GeV}$ analysis, since it is the nominal Booster extraction energy.

The described procedure was also utilized for analysis of both pulsed and DC septa (both in Booster extraction and Storage Ring injection), since all these magnets are stand-alone bends.

Figure 10 illustrates the tracking procedure on example of bend B1. The results of performed analysis are summarized in Table 5. 


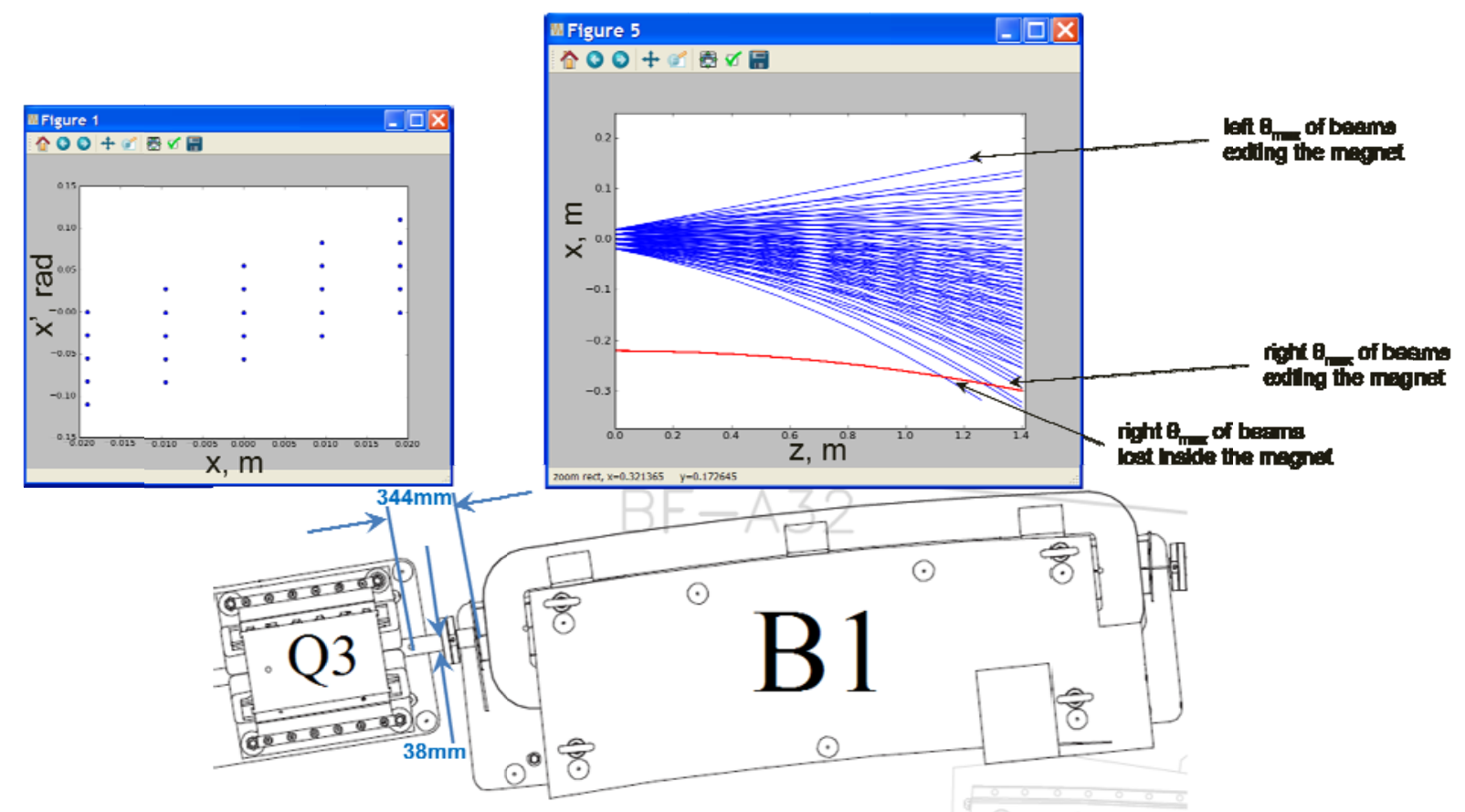

Figure 10: The phase space available to the beam centroids at the entrance of B1 (left plot) is defined by the geometric acceptance of Q3-B1 drift (its horizontal dimensions are given on the lower schematics). The phase space is filled with 25 beam centroids (for the clarity of the picture we reduced the number of beam centroid usually used in tracking by a factor of 100). Each of these beams is tracked through B1 (right plot) at its minimum, nominal and maximum settings. The red line on the right plot represents B1 yoke.

\section{References}

[1] BR-PH-LAT-0003 (REV B)

[2] NSLS-II Booster Final Design Report

[3] G. Wang, BSR lattice file

[4] Private communications with G. Ganetis

[5] Private communications with R. Fliller

[6] Private communications with B. Wahl

[7] Private communications with M. Johansen 


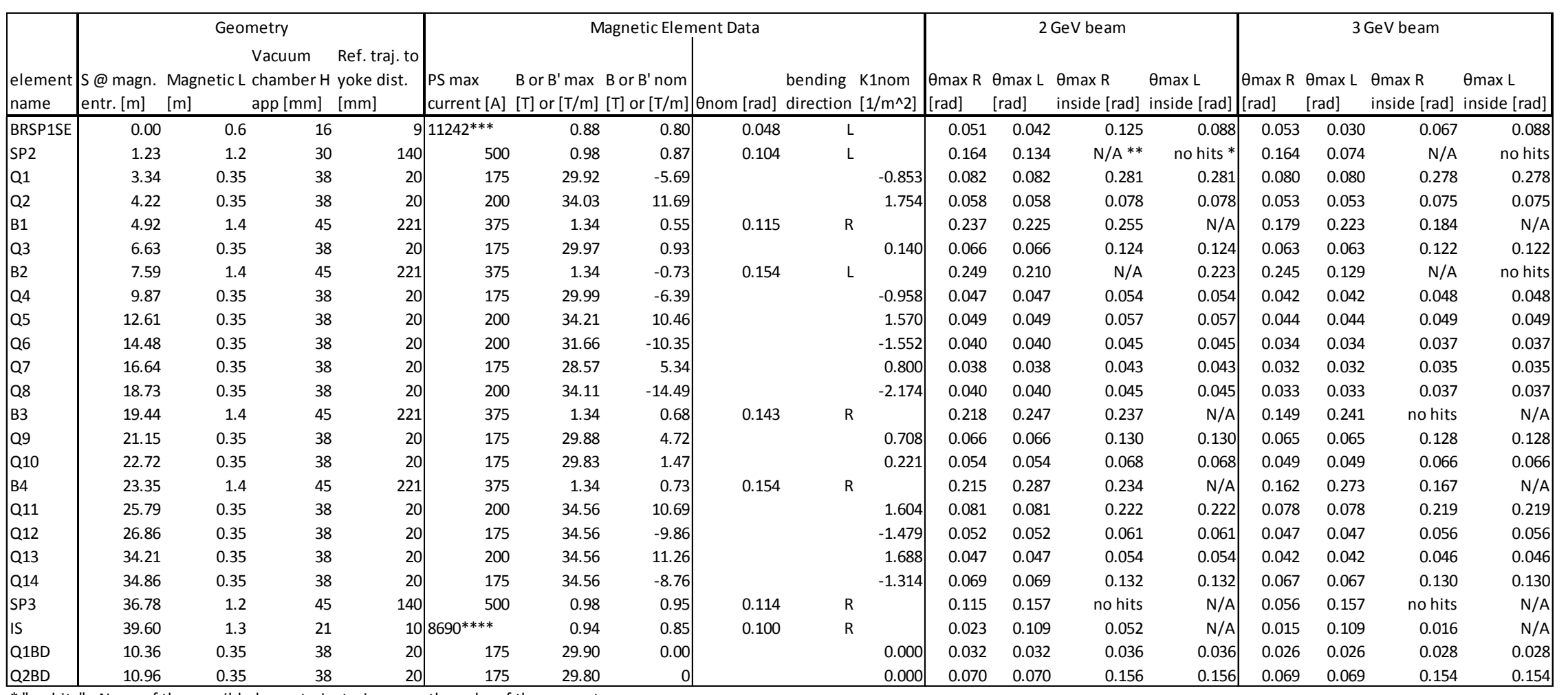

* "no hits" - None of the possible beam trajectories cross the yoke of the magnet

** "N/A" - No magnet yoke in this direction

*** Nominal $10220[\mathrm{~A}]+10 \%$

$* * * *$ Nominal $7900[\mathrm{~A}]+10 \%$

1.) "R" stands for deflection to the right w.r.t. beam direction, "L" stands for deflection to the left w.r.t. beam direction

2.) All deflection angles are given w.r.t. beam direction downstream of the magnet

Table 5: Maximum angles of lost beams and beams exiting the BSR magnets at $2 \mathrm{GeV}$ and $3 \mathrm{GeV}$. The $12^{\text {th }}, 13^{\text {th }}, 16^{\text {th }}$ and $17^{\text {th }}$ columns give angles at magnet exit. The $14^{\text {th }}, 15^{\text {th }}, 18^{\text {th }}$ and $19^{\text {th }}$ columns give angles of the beams lost inside the magnet. 